\title{
The role of human ADA2a in the regulation of p53 acetylation and stability
}

\author{
HUANG Jing ${ }^{1}$, ZHANG Li $^{1}$, XIAO Lin ${ }^{1}$, XU LanJun ${ }^{1}$, HU FanLei ${ }^{1}$, SHAO WenWei ${ }^{1}$, LIU Wei ${ }^{1}$, \\ MO XiaoNing ${ }^{1}$, SHI TaiPing ${ }^{1,2} \&$ QIU XiaoYan ${ }^{1 *}$ \\ ${ }^{1}$ Laboratory of Medical Immunology, School of Basic Medical Science, Peking University Health Science Center, Beijing 100191, China; \\ ${ }^{2}$ Chinese National Human Genome Center, Beijing 100176, China
}

Received September 19, 2010; accepted November 26, 2010

\begin{abstract}
The tumor suppressor protein p53 is a well-known transcription factor that functions as a critical component of the genotoxic stress response via regulating the expression of effector proteins that control cellular fate following DNA damage. The human p300/CBP-associated factor (PCAF)-containing histone acetyltransferase (HAT) complex is important for the stability and activity of p53 via its acetylation. The human homolog of yeast alteration/deficiency in activation 2a (ADA2a) is a stable component of the human PCAF-containing HAT complex. In this study, we demonstrated that p53 and hADA2a physically interact with each other in human HEK 293T cells. Using overexpression and small interfering RNA-mediated knockdown, we demonstrated that hADA2a stabilizes p53 via promoting its acetylation at lysine 320 - a PCAF-dependent acetylation site. Furthermore, hADA2a can potentiate the transcriptional activity of $\mathrm{p} 53$ at the $B A X$ and $p 21$ promoters to induce cell apoptosis and cell cycle arrest. Overall, our results establish that hADA2a, a component of the PCAF-containing histone acetyltransferase co-activator complex, is a mediator of acetylation-dependent stabilization and activation of p53 in mammalian cells.
\end{abstract}

hADA2a, p53, acetylation, PCAF

Citation: Huang J, Zhang L, Xiao L, et al. The role of human ADA2a in the regulation of p53 acetylation and stability. Chinese Sci Bull, 2011, 56: 397-405, doi: 10.1007/s11434-010-4311-0

The p53 tumor suppressor protein is a sequence-specific, DNA-binding transcription factor. In unstressed cells, p53 is maintained at a very low level, but it is rapidly stabilized and activated during periods of cellular stress, such as that induced by DNA damage. This results in the activation of multiple cellular processes including apoptosis and cell cycle checkpoints, via specific binding of p53 to the promoters of its target genes [1-4]. It has become clear that a cascade of post-translational modification, such as phosphorylation, ubiquitination, and acetylation, is required to stabilize p53 [5]. Acetylation of p53 provides a particularly important mechanism to control p53 stability as well as its activity $[3,6]$. p53 deacetylation by overexpression of histone deacetylase-associated proteins compromises its ability to

*Corresponding author (email: qiuxy@bjmu.edu.cn) induce cell cycle arrest and apoptosis [7]. In contrast, inhibition of p53 deacetylation increased p53's half-life and activity, suggesting that acetylation might play an important role in the turnover of the p53 protein [8]. The acetylation of p53 is dependent on the activity of the p300/CBP-associated factor (PCAF)-containing histone acetyltransferase (HAT) complex, which acetylates p53 at lysine K317R Notably, analysis of knock-in mice with a missense mutation (K317R) in the PCAF has suggested a negative regulatory role for the PCAF-dependent K317 (K320 in human p53) acetylation site $[9,10]$.

The PCAF-containing complex consists of more than 20 distinct polypeptides, including the transcriptional adaptor ADA2a. The ADA (human homolog of yeast alteration/deficiency in activation) genes (encoding ADA1, ADA2, ADA3/NGG1, general control non-derepressible 5 
(GCN5)/ADA4, and SPT20/ADA5) were genetically defined in the yeast Saccharomyces cerevisiae [11]. Yeast ADA proteins play a role in transcriptional initiation site selection, interact with basal transcription factors, and facilitate the acetylation of nucleosomal histones as components of ADA/GCN5 HAT complexes, which have multiple and distinct functions leading to gene-specific transcriptional activation [12]. It has been suggested that ADA2 plays an essential role in these complexes by participating in the recruitment of basal transcription factors, stabilizing the interactions with acidic activation domains, and potentiating the HAT activity of GCN5 [13-15]. Recently, several groups have reported that the Drosophila genome contains 2 distinct genes encoding ADA2 homologs: ADA2a and ADA2b. Biochemical characterization of the 2 ADA2 proteins demonstrated that both interact with the HAT/ GCN5 complex and participate in transcriptional activation [16]. However, functional differences between the 2 human ADA2 proteins, hADA2a and hADA2b, have been recently reported [17]. hADA2a, but not hADA2b, was shown to be a stable component of the human PCAF-con- taining HAT complex. However, it is still unclear how hADA2a functions in the PCAF-containing complex affect the transcriptional activity of p53.

In this study, we demonstrated that hADA2a physically interacted with p53 using coimmunoprecipitation (co-IP) and glutathione- $S$-transferase (GST) pull-down of overexpressed hADA2. Furthermore, given the ability of the hADA2a-containing PCAF complex to acetylate p53, we identified an important role for hADA2a in p53 acetylation, p53 stability, and p53 transcriptional activation following DNA damage, using hADA2a overexpression and small interfering RNA (siRNA)-mediated knockdown of endogenous hADA2a. These studies underscore the role of hADA2a in regulating $\mathrm{p} 53$ function.

\section{Materials and methods}

\subsection{Antibodies}

Monoclonal antibodies against p53 (DO1) and green fluorescent protein (GFP) were purchased from Medical \& Biological Laboratories Co., Ltd. (Naka-ka Nagoya, Japan), anti-acetylated p53 (K320) antiserum was obtained from Alpha Diagnostic (San Antonio, TX), a polyclonal antibody against p53 and a monoclonal antibody against p21 were purchased from Santa Cruz Biotechnology, Inc. (Santa Cruz, CA). Anti-GST and anti- $\beta$-actin antibodies were from Sigma (St. Louis, MO), the anti-hADA2a antibody was from Novus Biologicals (Littleton, CO), and IRDye ${ }^{\mathrm{TM}}$ 800-conjugated secondary antibodies against mouse IgG, rabbit IgG, streptavidin, or GFP were purchased from LI-COR Bioscience (Lincoln, NE). The acetyl-histone antibody sampler kit was purchased from Cell Signaling Technology (Lrvine, CA).

\subsection{Cell lines and transfections}

The human embryonic kidney cell line HEK 293T, a kind gift from Prof. Matsuda T., Japan) and the human osteosarcoma epithelial cell line U-2 OS (from ATCC) cells were maintained in Dulbecco's modified Eagle's medium (Invitrogen) supplemented with $10 \%$ fetal bovine serum (Biochrom, Berlin, Germany) and $L$-glutamine ( $2 \mathrm{mmol} / \mathrm{L})$ at $37^{\circ} \mathrm{C}$ in a humidified $5 \% \mathrm{CO}_{2}$ atmosphere. DNA transfection was performed using VigoFect (Vigorous, USA), a non-liposomal cationic formula, according to the manufacturer's instructions, or by electroporation with a single pulse of $120 \mathrm{~V}, 20 \mathrm{~ms}$, with $10 \mu \mathrm{g}$ plasmid/10 $/ 0^{6}$ cells in 2-mm gap cuvettes using an ECM 830 Square Wave Electroporation System (BTX, USA).

\subsection{Immunoprecipitation (IP), GST pull-down and immunoblotting}

HEK 293 T cells were transfected with different plasmids. The cells were harvested $36 \mathrm{~h}$ later and cell lysates were prepared with RIPA buffer (50 mmol/L HEPES pH 7.4, 50 $\mathrm{mmol} / \mathrm{L} \mathrm{NaCl}, 2 \mathrm{mmol} / \mathrm{L}$ EDTA, $2 \mathrm{mmol} / \mathrm{L}$ EGTA, 0.1\% SDS, $1 \%$ NP-40, with freshly added protease inhibitor cocktail (Roche). Cell lysates were precleared with $15 \mu \mathrm{L}$ of protein G beads (sigma, USA) (50\% slurry) for $1 \mathrm{~h}$ at $4^{\circ} \mathrm{C}$. For IP, $2 \mathrm{mg}$ of precleared supernatant was incubated with 1 $\mu \mathrm{g}$ of antibody for $2 \mathrm{~h}$ with occasional rocking. Next, $20 \mu \mathrm{L}$ of protein $\mathrm{G}$ beads were added to the above samples and rocked for an additional $2 \mathrm{~h}$ at $4^{\circ} \mathrm{C}$. The beads were washed 5 times with RIPA buffer, and the bound proteins were eluted and boiled with sample buffer. The supernatants were subjected to SDS-polyacrylamide gel electrophoresis (PAGE) and immunoblotting, as described previously [18]. The protein bands were visualized using an IRDye ${ }^{\mathrm{TM}}$ 800-conjugated secondary antibody; signals were detected on an Odyssey Infrared Imager (Li-COR Bioscience).

For GST pull-down, the GST-p53 and GST plasmids were transformed into E. coli strain BL21 (DE3). Expression of the fusion protein was induced by the addition of isopropyl $\beta$-D-1-thiogalactopyranoside to a final concentration of $1 \mathrm{mmol} / \mathrm{L}$, and incubation at $37^{\circ} \mathrm{C}$ for $3 \mathrm{~h}$. After lysis of the bacterial cells with lysozyme, GST or GST-p53 fusion protein was immobilized on glutathione-Sepharose beads (Amersham) according to the manufacturer's instructions. The beads were washed five times with ice-cold phosphate-buffered saline (PBS). The hADA2a protein was translated in vitro from the hADA2a-myc plasmid and labeled with biotin using a TNT T7 Quick Coupled transcription/translation system (Promega, USA). Fifty microliters of the product were incubated with the beads at $4{ }^{\circ} \mathrm{C}$ overnight. The beads were washed 6 times with ice-cold PBS, and then the bound protein was subjected to SDS-PAGE followed by immunoblotting with appropriate antibodies. 


\subsection{Immunofluorescence}

U-2 OS cells were plated on glass coverslips, then after $24 \mathrm{~h}$ they were fixed in PBS supplemented with $4 \%$ paraformaldehyde for $10 \mathrm{~min}$ at $4^{\circ} \mathrm{C}$, and permeabilized in PBS supplemented with $0.1 \%$ Triton X-100 for 30 min. After a 30 -min incubation in blocking buffer (2\% bovine serum albumin in PBS), the cells were incubated with rabbit anti-p53 and mouse anti-hADA2a antibodies at $4{ }^{\circ} \mathrm{C}$ overnight, then incubated with DyLight 488-conjugated goat anti-rabbit IgG and TRITC-labeled anti-mouse IgG (Jackson ImmunoResearch Laboratories, Inc, West Grove, PA, USA) for 1 h. 4',6-diamidino-2-phenylindole (DAPI; Sigma) was used to counterstain the nuclei. After several washing steps by $0.01 \mathrm{~mol} / \mathrm{L}$ PBS, the cells were observed under a Zeiss LSM 510 Meta system (Zeiss, Germany).

\subsection{RNA interference and real-time polymerase chain reaction analysis}

A specific siRNA against hADA2a (si-hADA2a; target sequence 5'-GAAUACAGGACAGCAGGCA-3') was chemically synthesized and PAGE-purified free of RNase contamination according to the manufacturer's instructions (Genechem Corporation, China). The non-silencing, control siRNA (si-NON) was confirmed to have no matches in the human genome by a BLAST search at the National Center for Biotechnology Information (www.ncbi.nlm.nih.gov). All siRNAs were dissolved to a concentration of $20 \mathrm{mmol} / \mathrm{L}$ in buffer containing $20 \mathrm{mmol} / \mathrm{L} \mathrm{KCl}, 6 \mathrm{mmol} / \mathrm{L}$ HEPES $\mathrm{pH} 7.5$ and $0.2 \mathrm{mmol} / \mathrm{L} \mathrm{MgCl}_{2}$. U-2 OS cell density was adjusted to $1 \times 10^{6} / 300 \mu \mathrm{L}$ and the indicated amount of siRNA was electroporated into cells. Total RNA was extracted using Trizol reagent (Life Technologies, Inc.). cDNAs were synthesized using a reverse transcriptase cDNA synthesis kit (Fermentas, Canada) with total RNA as the template. The cDNA were then subjected to reverse-transcription polymerase chain reaction (PCR) and real-time PCR analysis. The PCR primers used in this study were as follows: hADA2a forward: 5'-GCTCTGAAGATGGCTGTGGT-3' and hADA2a reverse: 5'-CTTGGAGCCTCTTGATTTC-3'; p53 forward: 5'-CTGCTCAGATAGCGATGGTC-3' and p53 reverse: 5'-CAGCTCTCGGAACATCTCG-3'; p21 forward: 5'-CCCGTGAGCGATGGAAC-3' and p21 reverse: 5'-CGGCGTTTGGAGTGGTAG-3'. For the internal control glyceraldehyde-3-phosphate dehydrogenase (GAP$\mathrm{DH})$ primers, the forward primer was 5'-CCACCCATGGCAAATTCCATGGCA-3' and the reverse primer was 5'-TCTAGACGGCAGGTCAGGTCAGGTCCACC-3'. PCR products were separated by $1.0 \%$ agarose gel electrophoresis and visualized by ethidium bromide staining.

\subsection{Analysis of p53 stability}

For the in vivo p53 degradation assay, 80\%-90\% confluent cultures of U-2 OS cells transfected transiently with hADA2a or si-hADA2a were incubated with cycloheximide $(100 \mu \mathrm{g} / \mathrm{mL})$. After 30,60 or $90 \mathrm{~min}$, the cells were harvested and immunoblotted using the anti-p53 antibody (DO-1). The intensity of the bands was quantified by densitometry.

\subsection{In vivo acetylation assay}

Cells were washed twice with ice-cold PBS and then grown in complete medium supplemented with the deacetylase inhibitor trichostatin A (TSA; Sigma) at a final concentration of $10 \mathrm{mmol} / \mathrm{L}$. Four hours after the addition of TSA, cells were washed twice with ice-cold PBS and lysed on ice in ice-cold lysis buffer $(50 \mathrm{mmol} / \mathrm{L}$ Tris $\mathrm{pH} 8.0,300$ $\mathrm{mmol} / \mathrm{L} \mathrm{NaCl}, 10 \mathrm{mmol} / \mathrm{L} \mathrm{MgCl}_{2}, 2.5 \mathrm{mmol} / \mathrm{L} \mathrm{CaCl}_{2}, 0.4 \%$ NP-40,) supplemented with $5 \mu \mathrm{mol} / \mathrm{L}$ TSA and protease inhibitor cocktail (Roche (China) Ltd). To detect acetylated human p53, equal amounts of cell lysates (250-500 $\mu \mathrm{g}$ protein) were incubated with $1 \mu \mathrm{g}$ of protein $\mathrm{G}$ agarose-immobilized antibody specific to human p53 (DO-1) for $2 \mathrm{~h}$ at $4^{\circ} \mathrm{C}$. The amount of p53 in the immunoprecipitate was estimated by western blotting using a rabbit polyclonal anti-p53 antibody. To determine the acetylation status of p53 in these cells, the same immunoprecipitates, normalized for the level of p53, were subjected to SDS-PAGE. Acetylated p53 was detected with rabbit anti-acetylated p53 (K320) antiserum by Western blotting.

\subsection{Reporter gene assay}

The luciferase reporter plasmid containing p21 binding sites (p21-Luc; Stratagene) and internal control pRL-TK (HSVthymidine kinase promoter, Promega) were co-transfected with the hADA2a plasmid or the specific si-hADA2a described above. Forty hours later, cells were assayed for luciferase activity. Luciferase activity was measured using the Luciferase Assay System (Vigorous, USA) according to the manufacturer's protocol.

\subsection{Flow cytometry}

Phosphatidylserine externalization analysis was performed as described previously [19]. Briefly, transfected cells $\left(2 \times 10^{5}\right)$ were trypsinized, washed twice with PBS, and resuspended in $200 \mu \mathrm{L}$ binding buffer $(10 \mathrm{mmol} / \mathrm{L}$ HEPES $\mathrm{pH}$ 7.4, $140 \mathrm{mmol} / \mathrm{L} \mathrm{NaCl}, 1 \mathrm{mmol} / \mathrm{L} \mathrm{MgCl}_{2}, 5 \mathrm{mmol} / \mathrm{L} \mathrm{KCl}$, $2.5 \mathrm{mmol} / \mathrm{L} \mathrm{CaCl}_{2}$ ). Fluorescein isothiocyanate-conjugated Annexin V (Beijing Biosea, China) was added to a final concentration of $0.5 \mu \mathrm{g} / \mathrm{mL}$. After incubation for $30 \mathrm{~min}$ at room temperature, propidium iodide (PI) was added at 1 $\mu \mathrm{g} / \mathrm{mL}$, and the samples were immediately analyzed on a FACSCalibur flow cytometer (Becton Dickinson, San Jose, CA). 
For cell cycle analysis, transfected U-2 OS cells $\left(5 \times 10^{5}\right)$ were fixed in $70 \%(\mathrm{v} / \mathrm{v})$ ethanol overnight at $4^{\circ} \mathrm{C}$. After washing with PBS, cells were incubated with RNase A $(0.5$ $\mathrm{mg} / \mathrm{mL}$; Sigma) at $37^{\circ} \mathrm{C}$ for $30 \mathrm{~min}$. Finally, after staining with PI $(50 \mu \mathrm{g} / \mathrm{mL})$, the DNA content per cell was measured using the CellQuest Pro program on the FACSCalibur flow cytometer (Becton Dickinson), and the data were analyzed using ModFit software.

\section{Results}

\section{1 hADA2a interacts with p53}

To confirm the interaction of hADA2a and p53 in mammalian cells, Flag-p53 and hADA2a-GFP plasmids were co-transfected into HEK $293 \mathrm{~T}$ cells. After $30 \mathrm{~h}$, the cell lysates were subjected to IP with an anti-GFP antibody. Western blotting revealed that Flag-p53 co-immunoprecipitated with hADA2a-GFP (Figure 1(a)), demonstrating that the two proteins exist in a complex in HEK 293T cells.

We further investigated the binding of hADA2a to p53 by GST pull-down assays. As expected, the full-length form of hADA2a, generated and labeled with biotin using in vitro transcription/translation, was pulled down by a recombinant GST-p53 fusion protein (Figure 1(b)). In contrast, the control
GST protein showed no interaction.

To confirm the hADA2a-p53 association, we performed co-localization assays. U-2 OS cells were plated for $24 \mathrm{~h}$, then treated with or without UV irradiation $\left(20 \mathrm{~J} / \mathrm{m}^{2}\right)$ to induce DNA damage, and incubated with mouse antihADA2a and rabbit anti-p53 antibodies. As shown previously, the same nuclear localization of endogenous ADA2a was detected in U-2 OS cells without UV irradiation as that in HEK 293T cells [20]. Moreover, hADA2a and p53 partly co-localized and showed a relatively diffuse nuclear distribution pattern in U-2 OS cells with or without UV irradiation (Figure 1(c)). All the above results support our findings of interaction between hADA2a and p53.

\subsection{Ectopic hADA2a expression stabilizes p53}

Because hADA2a bound to p53, we assessed whether hADA2a influenced endogenous p53 at the mRNA or protein level. The hADA2a-GFP plasmid was transfected into U-2 OS cells, and then parallel samples were processed for RT-PCR and western blotting. RT-PCR showed that the mRNA level of $p 53$ remained unchanged, similar to that of the housekeeping gene GAPDH used as a loading control (Figure 2(a)). Western blotting indicated that overexpression of hADA2a increased p53 protein levels (Figure 2(b)).
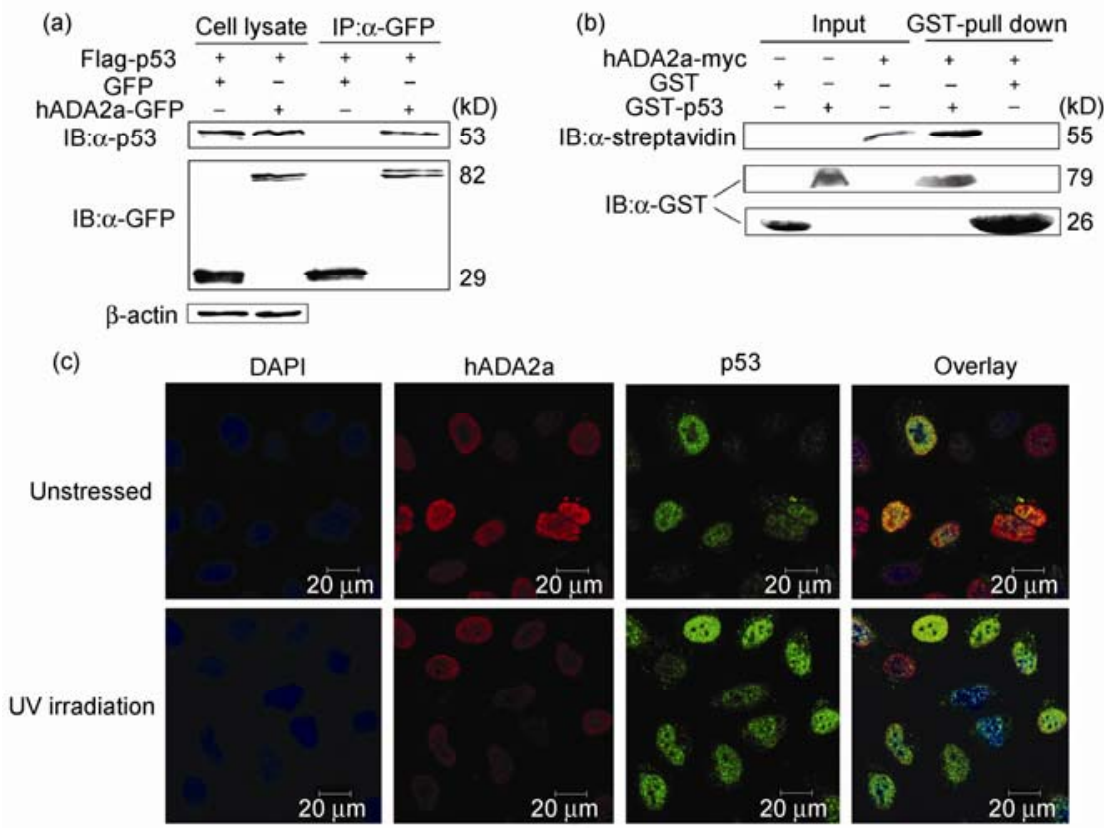

Figure 1 hADA2a directly interacts with p53. (a) Co-immunoprecipitation of hADA2a and p53 in HEK 293T cells. Cells were co-transfected with Flag-p53 and hADA2a-GFP or GFP alone as a control. Total cell extracts were immunoprecipitated using an anti-GFP antibody as indicated. Immunoprecipitated (IP) proteins were analyzed for the presence of p53 by immunoblotting (IB), using IRDye ${ }^{\mathrm{TM}} 800$-conjugated antibody against GFP to detect the immunoprecipitated hADA2a-GFP, or GFP proteins as the transfection control. (b) GST pull-down assay of hADA2a and GST-p53. Biotin-labeled hADA2a protein generated by in vitro transcription/translation was pulled down by GST-p53 fusion protein and GST protein. Proteins pulled down were analyzed for biotin-labeled hADA2a using IRDye ${ }^{\mathrm{TM}} 800$-conjugated antibody against streptavidin. One microliter of labeled translated hADA2a protein, $1 \mu \mathrm{g}$ of GST-p53 fusion protein or $1 \mu \mathrm{g}$ of GST protein were used as input. (c) hADA2a partly colocalized with p53 in U-2 OS cells treated with or without UV irradiation (20 $\mathrm{J} / \mathrm{m}^{2}, 12 \mathrm{~h}$ ). Immunofluorescence was performed as described. Endogenous hADA2a expression was detected with mouse anti-hADA2a antibody followed by TRITC-labeled anti-mouse secondary antibody; endogenous p53 was detected by rabbit anti-p53 antibody followed by DyLight 488 -conjugated anti-rabbit secondary antibody. Cell nuclei were stained with DAPI. 


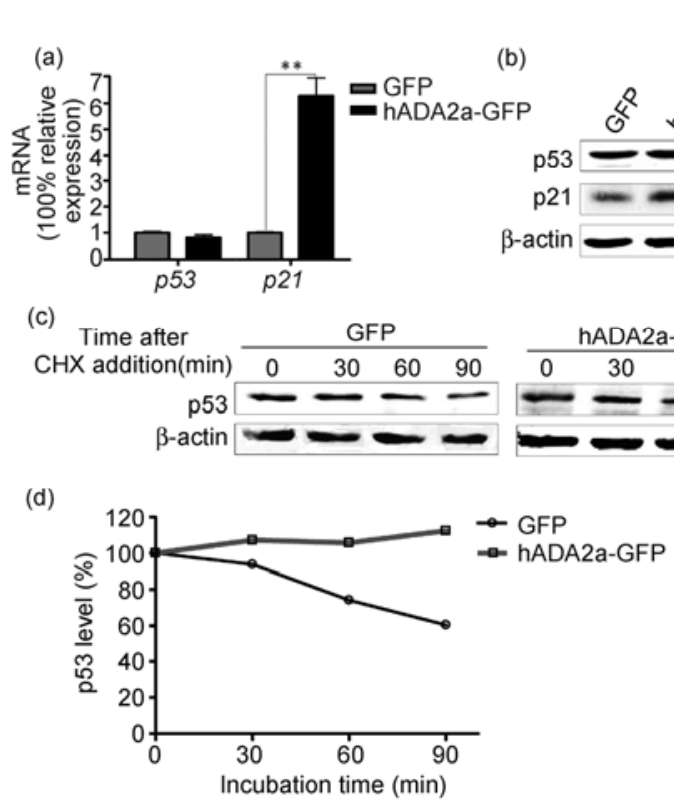

Figure 2 hADA2a stabilizes p53 protein by post-translational modification. U-2 OS cells were transfected with the indicated plasmids for $48 \mathrm{~h}$. Cells were then harvested for protein or mRNA analysis. (a) U-2 OS cells were analyzed to detect the levels of $p 53, p 21$, or $G A P D H$ (loading control) by real-time PCR. Data are presented as the mean \pm SD. **, $P<0.01$. (b) Equal amounts of whole cell extracts were immunoblotted using anti-p53, anti-p21, or anti- $\beta$-actin antibodies. (c) hADA2a overexpression extended the half-life of $\mathrm{p} 53$. The transfected cells were treated with cycloheximide $(\mathrm{CHX} ; 100 \mu \mathrm{g} / \mathrm{mL})$ and harvested at the indicated times. Cell lysates were analyzed by immunoblotting using anti-p53 or anti- $\beta$-actin antibodies. (d) The intensity of p53 relative to $\beta$-actin was quantified by densitometry using Li-Cor Odyssey software, and plotted against the time of cycloheximide treatment.

In contrast, $\mathrm{p} 21$, a protein functionally downstream of $\mathrm{p} 53$, was significantly increased at both the mRNA and the protein levels (Figure 2(a) and (b)). These observations indicate that the accumulation of p53 protein upon hADA2a overexpression was likely because of post-translational modification.

To investigate whether the hADA2a-dependent increase in p53 protein reflected an increase in its stability, we assessed the half-life of p53 following a cycloheximide block of new protein synthesis. U-2 OS cells were transfected with hADA2a-GFP or GFP alone. The transfected cells were incubated with cycloheximide, and the level of p53 was assessed by immunoblotting. As shown in Figure 2(c) and $(\mathrm{d})$, overexpression of hADA2a resulted in a slower loss of p53 signal compared with that in cells without hADA2a overexpression. This suggests that ectopic hADA2a expression stabilizes endogenous p53.

\section{3 siRNA-mediated depletion of hADA2a destabilizes p53}

To test the role of hADA2a in stabilizing p53, a specific siRNA for $h A D A 2 a$ was designed to silence the expression of endogenous hADA2a in U-2 OS cells. We found that hADA2a mRNA and protein levels were significantly decreased in cells transfected with si-hADA2a (Figure 3(a)). As illustrated in Figure 3(b) and (c), endogenous p53 protein, but not p53 mRNA, was significantly decreased. Moreover, p21 was also decreased at both the mRNA and protein levels.

We next assessed the half-life of p53 protein after si-hADA2a transfection. As shown in Figure 3(d) and (e), we determined that depletion of endogenous hADA2a was associated with a slight acceleration in p53 decay. This suggests that the decrease in p53 protein after hADA2a knockdown was because of destabilization of the p53 protein.

\section{4 hADA2a affects the level of p53 by participating in the PCAF complex}

To determine the role of hADA2a in the PCAF complex, we
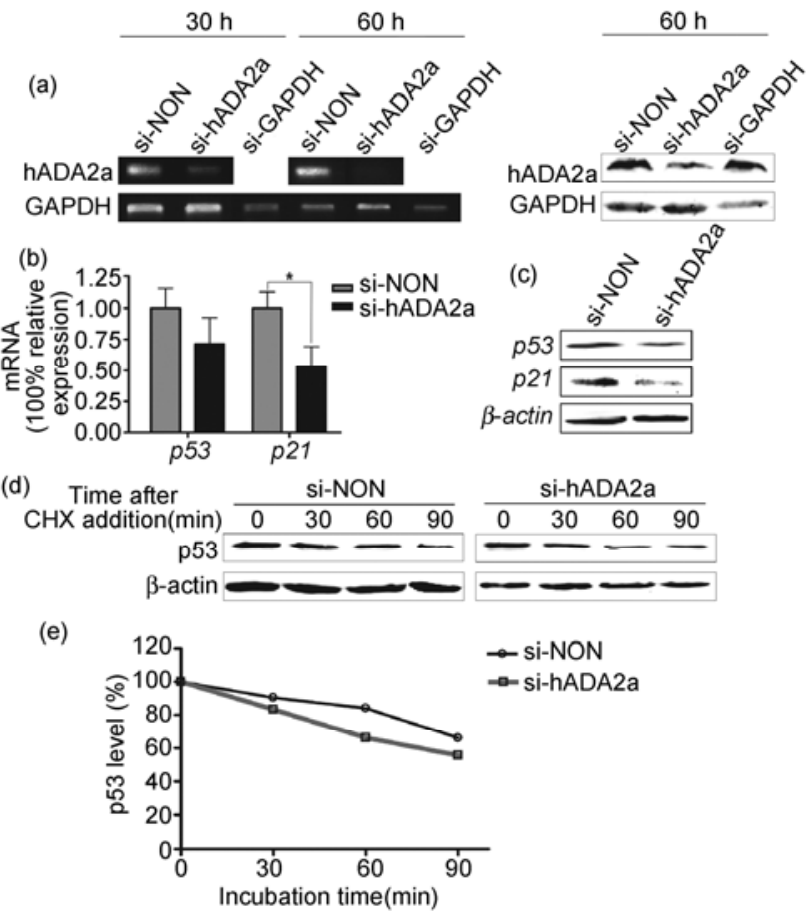

Figure 3 Transient knockdown of hADA2a expression decreases the level of endogenous p53. U-2 OS cells were transfected with specific siRNA against $h A D A 2 a$ (si-hADA2a), control siRNA (si-NON) or siRNA against GAPDH (si-GAPDH). Cells were harvested at different time points for protein or mRNA analysis. (a) RT-PCR results for $h A D A 2 a$ mRNA expression and immunoblotting results for hADA2a protein expression. si-hADA2a showed a strong inhibitory effect on hADA2a mRNA and protein expression in U-2 OS cells $60 \mathrm{~h}$ after transfection with si-hADA2a compared with si-NON. (b) Total RNAs were analyzed by real-time PCR to detect the levels of $p 53, p 21$, or $G A P D H$ as a loading control. Data are presented as the mean $\pm \mathrm{SD}$. ${ }^{*}, P<0.05$. (c) Equal amounts of protein from cell lysates were subjected to immunoblotting using anti-p53, anti-p21, or anti- $\beta$-actin antibodies. (d) Knockdown of endogenous hADA2a accelerated the decay of endogenous p53. Transfected cells were treated with cycloheximide $(100 \mu \mathrm{g} / \mathrm{mL})$ and harvested at the indicated times. Total cell lysates were analyzed for the presence of p53 by immunoblotting. (e) Relative p53 protein levels were quantified by densitometry against $\beta$-actin using Li-Cor Odyssey software and plotted against the time of cycloheximide treatment. 
first tested the interaction between hADA2a and PCAF using co-IP. After transient transfection in HEK 293T cells with pCMV-Tag2B-PCAF (Flag-tagged) and hADA2aGFP, the proteins were immunoprecipitated with anti-Flag or anti-GFP antibody, followed by western blotting with anti-GFP or anti-Flag antibody. As shown in Figure 4(a), a specific band corresponding to hADA2a or PCAF was observed in cell lysates co-transfected with pCMV-Tag2BPCAF or hADA2a-GFP, whereas no band was observed in the cell lysates transfected with the empty vector (negative control). This confirmed the interaction between PCAF and hADA2a.

To address whether hADA2a promotes acetylation of p53 at K320, U-2 OS cells were transfected with the hADA2a plasmid or si-hADA2a. After $24 \mathrm{~h}$, the transfected cells were UV irradiated at $20 \mathrm{~J} / \mathrm{m}^{2}$ for $16 \mathrm{~h}$ to induce DNA damage, and then the levels of acetylated p53 were detected using IP and immunoblotting. As illustrated in Figure 4(b), the levels of acetylated p53 at K320 were significantly higher in cells overexpressing hADA2a $(P<0.05)$, and significantly lower in cells transfected with si-hADA2a $(P<$ $0.05)$.

\section{5 hADA2a potentiates p53-induced apoptosis}

We next investigated whether hADA2a was required for apoptosis induced by DNA damage. U-2 OS cells were transfected with either pcDB-hADA2a or si-hADA2a, and then were treated with $20 \mathrm{~J} / \mathrm{m}^{2}$ of UV irradiation. After $24 \mathrm{~h}$, apoptosis was monitored by caspase-mediated poly (ADPribose) polymerase (PARP) cleavage and BAX expression by western blotting. Overexpressed hADA2a increased PARP cleavage and BAX expression compared with the vector control, independent of UV irradiation (Figure 5(a)).
In contrast, si-hADA2a inhibited PARP cleavage and BAX expression slightly after UV irradiation (Figure 5(b)).

Apoptotic cells were also assessed by Annexin-V/PI labeling flow cytometry. As shown in Figure 5(c), overexpression of hADA2a alone significantly induced apoptosis, showing a $20 \%$ increase compared with the control plasmid $(P<0.05)$. However, we did not observe a synergistic effect between hADA2a overexpression and UV irradiation. si-hADA2a slightly reduced UV-triggered apoptosis.

\section{6 hADA2a increases p53-mediated transactivation of p21 and induces a cell cycle checkpoint}

We determined whether hADA2a influences p53 activity in promoting p21 expression in U-2 OS cells. U-2 OS cells were transiently co-transfected with the hADA2a plasmid or si-hADA2a and a p21 luciferase reporter construct, together with pRL-TK, then the luciferase activity was determined. Overexpressed hADA2a resulted in an approximately 2-fold increase in p53-mediated transcriptional activation of p21 compared with the vector control. Knockdown of endogenous hADA2a strongly inhibited p53-mediated transcriptional activation of $\mathrm{p} 21$ compared with the si-NON control (Figure 6(a)). We also examined whether hADA2a affected the cell cycle checkpoint, when cells respond to DNA damage. U-2 OS cells were transfected with either pcDBhADA2a or si-hADA2a. Then, they were treated with $20 \mathrm{~J} / \mathrm{m}^{2}$ of UV irradiation for $20 \mathrm{~h}$. In response to DNA damage induced by UV treatment, more hADA2a-overexpressing cells were in G1 than in S phase, and fewer hADA2a knockdown cells were in G1 than in S phase (Figure 6(b)). These results indicate that hADA2a might play an important role in the cell cycle checkpoint through p53-dependent p21 transactivation in response to DNA damage.
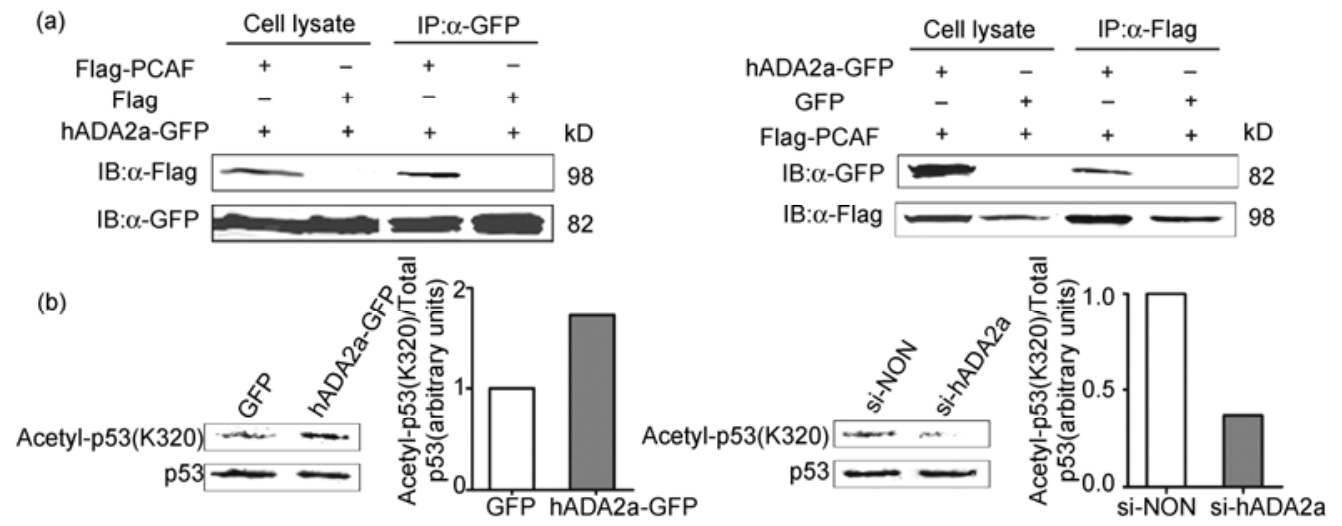

Figure 4 hADA2a participates in the PCAF complex and affects p53 acetylation. (a) Co-immunoprecipitation of hADA2a and PCAF in HEK 293T cells. Cells were co-transfected with Flag-PCAF and hADA2a-GFP or empty vector. Thirty-six hours after transfection, whole cell lysates were subjected to immunoprecipitation (IP) using anti-GFP or anti-Flag antibody as indicated. Immunoprecipitated proteins were then analyzed for the presence of PCAF or hADA2a by immunoblotting (IB), using IRDye ${ }^{\text {TM }}$ 800-conjugated antibody against GFP to detect the immunoprecipitated hADA2a-GFP, and mouse anti-Flag antibody to detect the immunoprecipitated Flag-PCAF. (b) U-2 OS cells were transfected with either hADA2a-GFP, GFP alone, si-NON or si-hADA2a. After $24 \mathrm{~h}$, the transfected cells were UV irradiated at $20 \mathrm{~J} / \mathrm{m}^{2}$ for $16 \mathrm{~h}$, cells were harvested, and equivalent amounts of cell lysates were immunoprecipitated using anti-p53 monoclonal antibody (DO-1). Then the immunoprecipitated proteins were analyzed for their levels of total and acetylated p53 using the indicated antibodies. Also, the intensity of acetylated p53 was quantitated and normalized to the total p53 signal using Image J software. The histograms show the ratios of acetylated versus total $\mathrm{p} 53$. 


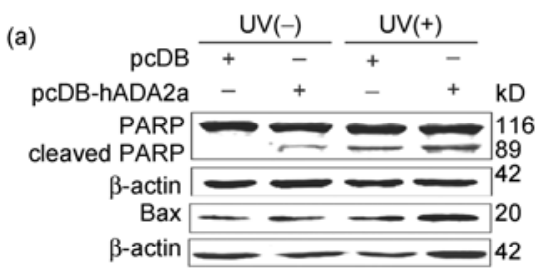

(c)
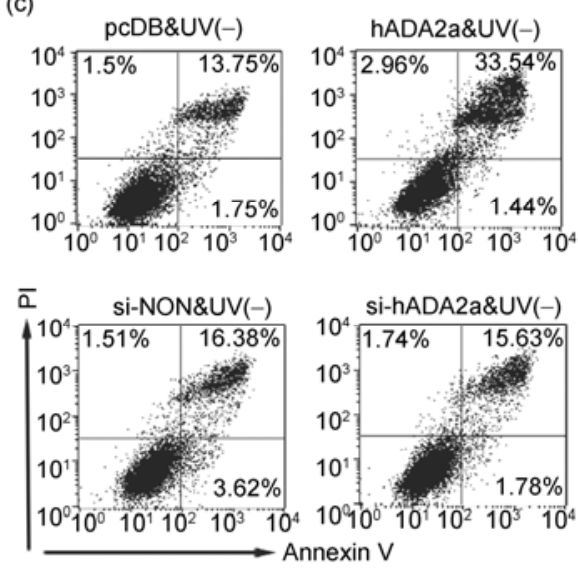

(b)
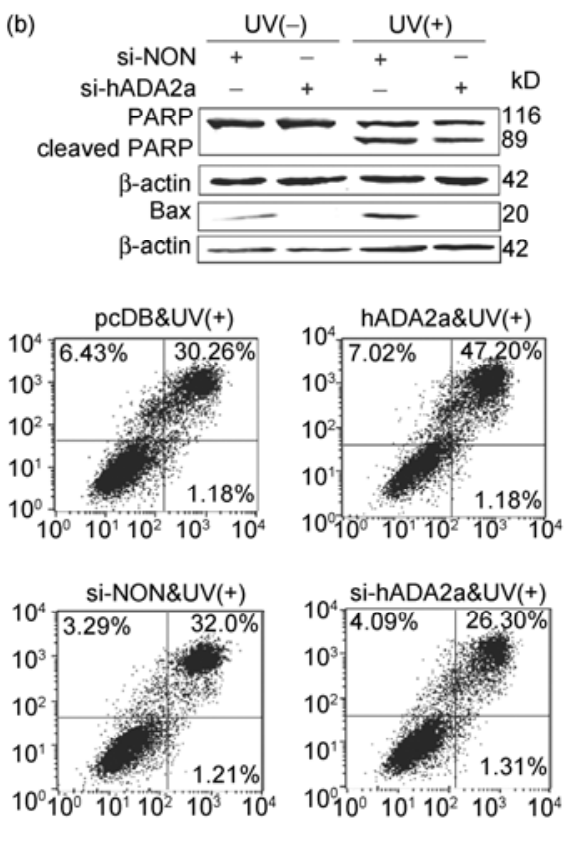

Figure 5 Effect of hADA2a on p53-induced apoptosis. U-2 OS cells were transfected with pcDB-hADA2a, pcDB, si-NON, or si-hADA2a. After 24 h, the cells were either mock-treated or treated with $20 \mathrm{~J} / \mathrm{m}^{2}$ of UV irradiation for another $24 \mathrm{~h}$. (a) and (b) Representative western blots showing the expression of proteins associated with apoptosis (PARP and BAX). Equal protein loading was confirmed by re-probing the membrane with $\beta$-actin. (c) Transfected U-2 OS cells were harvested and stained with Annexin V/PI. The percentage of Annexin V-and/or PI-positive cells is shown. Annexin V- ( $x$-axis) and PI- ( $y$-axis) double negative cells are viable. Annexin V-positive and PI-weakly positive cells are apoptotic (they have an increased cell membrane permeability), whereas Annexin V-positive and PI-strongly positive cells are late apoptotic/necrotic and PI-positive only cells are necrotic. The data are representative of three independent experiments.

(a)

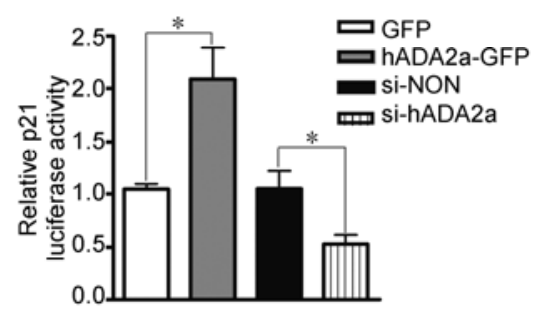

(b)

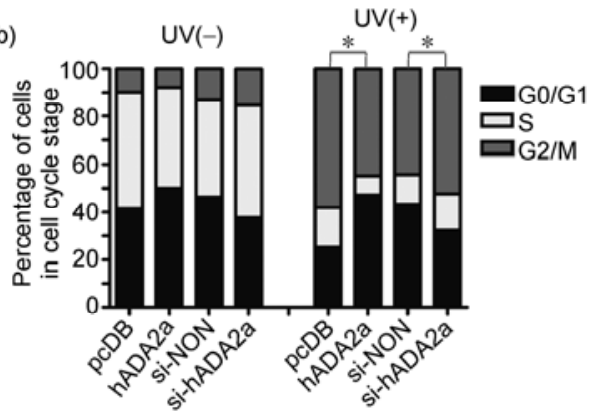

Figure 6 Effect of hADA2a on p53 transactivation of $p 21$ and the cell cycle checkpoint. (a) The effect of hADA2a in activating the $p 21$ promoter. Cells were co-transfected with hADA2a plasmid or si-hADA2a and a p21 luciferase reporter construct, together with pRL-TK. The data shown are relative luciferase activities normalized to Renilla luciferase activity, and are the means \pm SD of three experiments. (b) U-2 OS cells were transfected with either pcDB-hADA2a, pcDB, si-NON, or si-hADA2a. After $24 \mathrm{~h}, \mathrm{U}-2$ OS cells were either mock-treated or treated with $20 \mathrm{~J} / \mathrm{m}^{2}$ of UV irradiation for $20 \mathrm{~h}$. The cells were then harvested, stained with propidium iodide, and subjected to flow cytometry. ${ }^{*}, P<0.05$.

\section{Discussion}

p53 is a sequence-specific homotetrameric transcription factor involved in the transactivation of genes that are responsible for multiple cellular processes, such as apoptosis, cell cycle arrest, and DNA repair [21]. Accumulating evidence had indicated that post-translational modifications of $\mathrm{p} 53$, such as acetylation, play important roles in regulating p53 stability and activity. The role of p53 acetylation at multiple C-terminal lysine resides, mediated by the co-activators
PCAF and p300, has been extensively studied using biochemistry and cell transfection assays [9]. Here, we demonstrated an important role for hADA2a in the stabilization and activation of $\mathrm{p} 53$. We also provided evidence that the hADA2a-dependent regulation of p53 stability and activity is mediated by acetylation of p53 at K320 through participating in the PCAF complex. Furthermore, hADA2a potentiated the transcriptional activity of $\mathrm{p} 53$ at the $B A X$ and $p 21$ promoters, inducing apoptosis and cell cycle arrest.

ADA2 was initially identified as a core component of the 
yeast ADA co-activator complex, which associated with the HAT protein Gen5 [15]. No role for ADA2 independent of Gcn5 has been characterized in Saccharomyces cerevisiae, although multiple forms of ADA2 exist in Drosophila melanogaster [16,22], plants [23], mice, and humans [17]. In Drosophila, ADA2a and ADA2b are required for normal development but have distinct functions [24]. Recent studies of ADA2a and ADA2b in higher eukaryotes, including mammals, have demonstrated that these proteins assemble into multiple complexes that vary considerably. In addition, hADA3 also participates in these complexes and associates with p53; hADA3 overexpression increases the transcriptional activity of p53 by affecting p53 acetylation [25-27]. Here, we showed, using Co-IP, GST pull-down, and colocalization assays, that hADA2a also directly interacts with p53.

We found consistently that overexpression of hADA2a stabilized the p53 protein, while depletion of endogenous hADA2a was associated with a slight acceleration in p53 decay. However, the physiological implications of this interaction, and the mechanism of hADA2a-mediated regulation of p53 are still unclear. Recent studies have demonstrated that many proteins involved in transcriptional regulation, including transcription factors themselves, can be modified by acetylation. Specifically, acetylation of p53 provides a crucial mechanism to control its stability and activity. Two acetyltransferases, p300 and PCAF, have been implicated in promoting p53 C-terminal acetylation, which favors p53 stabilization [3,9]. Ogryzko et al. [28] identified that hADA2a was a stable component of the human PCAFcontaining HAT complex, was involved in the acetylation of p53 K320 and increased the target DNA-binding affinity of $\mathrm{p} 53$. Our results showed that hADA2a did indeed participate in the PCAF complex; moreover hADA2a affected acetylation of the PCAF-dependent acetylation site at K320 in $\mathrm{p} 53$. We suggest that hADA2a plays a crucial role in mediating p53 etylation by recruiting HATs.

We also looked for evidence that hADA2a plays a role in regulating p53 function and in modulating the cellular response to DNA damage. p53 induces apoptosis via upregulating several proapoptotic genes, such as $B A X$ [29]. We demonstrated that overexpression of hADA2a significantly induced apoptosis. Furthermore, Barlev et al. [8] indicated that acetylation of p53 and its association with co-activators was required for the physiological function of p53 in cell cycle arrest following DNA damage. The search for mediators of p53-dependent cell cycle arrest has identified many cellular p53 target genes, including p21 $1^{\text {cipl/wafl }}$, a well characterized cyclin-dependent kinase inhibitor, which mediates cell cycle arrest in G1 when overexpressed [30]. Our results showed that hADA2a potentiated the transcriptional activity of p53 at the $p 21$ promoter and led to a p53-dependent cell cycle arrest in response to UV irradiation. Our data indicate that hADA2a affects the acetylation of p53, which is responsible for the regulation of pro-apoptotic and cell cycle arrest-promoting gene expression after DNA damage. Thus, we suggest that hADA2a plays a major role in the acetylation-dependent stabilization and activity of p53. This represents a major step forward in understanding the mechanisms that control p53 function.

hADA2a contains a Swi3p, Rsc8p and Moira (SWIRM) domain that might function to potentiate chromosomal structural changes necessary for the recruitment of protein complexes for chromatin remodeling or transcription [20]. It has been suggested that hADA2a is a molecular scaffold for the histone acetyltransferases that promotes histone lysine acetylation and transcriptional activation. However, it remains to be determined how hADA2a interacts with p53 to regulate its acetylation. Further analysis of the role of hADA2a in the post-translational regulation of the $p 53$ gene might provide mechanistic insight into the regulation of p53-targeted genes that contribute to cellular apoptosis and the cell cycle.

In conclusion, our identification of the interaction between hADA2a and human p53, and our description of hADA2a as an important regulator of p53 acetylation, stability, and activity, has provided new insight into p53 biology. Further studies of hADA2a-deficient animals and p53-directed HATs should help delineate the biochemical basis as well as the overall biological importance of hADA2a-dependent p53 regulation.

This work was supported by the Ministry of Science and Technology for Drug Development (2009ZX09503-004), the National Natural Sciences Foundation of China (30901303) and Foundation for New Youth Scholars of Peking University Health Science Center (BMU20090459). We thank Prof. MA DaLong (Peking University Center for Human Disease Genomics) for his comments and discussion, and ZHANG Pei (Peking University Third Hospital, Beijing, China) for her excellent technical support.

1 Wahl G M, Carr A M. The evolution of diverse biological responses to DNA damage: Insights from yeast and p53. Nat Cell Biol, 2001, 3: E277-E286

2 Appella E, Anderson C W. Post-translational modifications and activation of p53 by genotoxic stresses. Eur J Biochem, 2001, 268: 2764-2772

3 Sakaguchi K, Herrera J E, Saito S, et al. DNA damage activates p53 through a phosphorylation-acetylation cascade. Genes Dev, 1998, 12: 2831-2841

4 Vogelstein B, Lane D, Levine A J. Surfing the p53 network. Nature, 2000, 408: 307-310

5 Bode A M, Dong Z. Post-translational modification of p53 in tumorigenesis. Nat Rev Cancer, 2004, 4: 793-805

6 Oren M. Regulation of the p53 tumor suppressor protein. J Biol Chem, 1999, 274: 36031-36034

7 Luo J, Su F, Chen D, et al. Deacetylation of p53 modulates its effect on cell growth and apoptosis. Nature, 2000, 408: 377-381

8 Barlev N A, Liu L, Chehab N H, et al. Acetylation of p53 activates transcription through recruitment of coactivators/histone acetyltransferases. Mol Cell, 2001, 8: 1243-1254

9 Liu L, Scolnick D M, Trievel R C, et al. p53 sites acetylated in vitro by PCAF and p300 are acetylated in vivo in response to DNA damage. Mol Cell Biol, 1999, 19: 1202-1209

10 Chao C, Wu Z, Mazur S J, et al. Acetylation of mouse p53 at lysine 317 negatively regulates p53 apoptotic activities after DNA damage. 
Mol Cell Biol, 2006, 26: 6859-6869

11 Berger S L, Pina B, Silverman N, et al. Genetic isolation of ADA2: A potential transcriptional adaptor required for function of certain acidic activation domains. Cell, 1992, 70: 251-265

12 Grant P A, Duggan L, Cote J, et al. Yeast Gen5 functions in two multisubunit complexes to acetylate nucleosomal histones: Characterization of an Ada complex and the SAGA (Spt/Ada) complex. Genes Dev, 1997, 11: 1640-1650

13 Anafi M, Yang Y F, Barlev N A, et al. GCN5 and ADA adaptor proteins regulate triiodothyronine/GRIP1 and SRC-1 coactivator-dependent gene activation by the human thyroid hormone receptor. Mol Endocrinol, 2000, 14: 718-732

14 Barlev N A, Candau R, Wang L, et al. Characterization of physical interactions of the putative transcriptional adaptor, ADA2, with acidic activation domains and TATA-binding protein. J Biol Chem, 1995, 270: 19337-19344

15 Balasubramanian R, Pray-Grant M G, Selleck W, et al. Role of the Ada2 and Ada3 transcriptional coactivators in histone acetylation. J Biol Chem, 2002, 277: 7989-7995

16 Muratoglu S, Georgieva S, Papai G, et al. Two different Drosophila ADA2 homologues are present in distinct GCN5 histone acetyltransferase-containing complexes. Mol Cell Biol, 2003, 23: 306-321

17 Barlev N A, Emelyanov A V, Castagnino P, et al. A novel human Ada2 homologue functions with Gen5 or Brg1 to coactivate transcription. Mol Cell Biol, 2003, 23: 6944-6957

18 Huang J, Shi T, Ma T, et al. CCDC134, a novel secretory protein, inhibits activation of ERK and JNK, but not p38 MAPK. Cell Mol Life Sci, 2008, 65: 338-349

19 Wang Y, Li X, Wang L, et al. An alternative form of paraptosis-like cell death, triggered by TAJ/TROY and enhanced by PDCD5 over- expression. J Cell Sci, 2004, 117: 1525-1532

20 Qian C, Zhang Q, Li S, et al. Structure and chromosomal DNA binding of the SWIRM domain. Nat Struct Mol Biol, 2005, 12: 1078-1085

21 Hofseth L J, Hussain S P, Harris C C. p53: 25 years after its discovery. Trends Pharmacol Sci, 2004, 25: 177-181

22 Kusch T, Guelman S, Abmayr S M, et al. Two Drosophila Ada2 homologues function in different multiprotein complexes. Mol Cell Biol, 2003, 23: 3305-3319

23 Vlachonasios K E, Thomashow M F, Triezenberg S J. Disruption mutations of ADA2b and GCN5 transcriptional adaptor genes dramatically affect Arabidopsis growth, development, and gene expression. Plant Cell, 2003, 15: 626-638

24 Pankotai T, Komonyi O, Bodai L, et al. The homologous Drosophila transcriptional adaptors ADA2a and $\mathrm{ADA} 2 \mathrm{~b}$ are both required for normal development but have different functions. Mol Cell Biol, 2005, 25: 8215-8227

25 Kumar A, Zhao Y, Meng G, et al. Human papillomavirus oncoprotein E6 inactivates the transcriptional coactivator human ADA3. Mol Cell Biol, 2002, 22: 5801-5812

26 Wang T, Kobayashi T, Takimoto R, et al. hADA3 is required for p53 activity. Embo J, 2001, 20: 6404-6413

27 Nag A, Germaniuk-Kurowska A, Dimri M, et al. An essential role of human Ada3 in p53 acetylation. J Biol Chem, 2007, 282: 8812-8820

28 Ogryzko V V K T, Zhang X, Schiltz R L, et al. Histone-like TAFs within the PCAF histone acetylase complex. Cell, 1998, 94: 35-44

29 Miyashita T R J. Tumor suppressor p53 is a direct transcriptional activator of the human bax gene. Cell, 1995, 80: 293-299

30 el-Deiry W S, Tokino T, Velculescu V E, et al. WAF1, a potential mediator of p53 tumor suppression. Cell, 1993, 75: 817-825

Open Access This article is distributed under the terms of the Creative Commons Attribution License which permits any use, distribution, and reproduction in any medium, provided the original author(s) and source are credited. 